\title{
Prolonged survival following everolimus combined with temozolomide for metastatic malignant melanoma with FBXW7 mutation: a case report and literature review
}

\author{
Bin Wang ${ }^{1,2,3 \#}$, Chao $\mathbf{Q u}^{1,2,3 \#}$, Qin Zhao ${ }^{1,2,3}$, Huanhuan Wang ${ }^{1,2,3}$, Jinlong Wei ${ }^{1,2,3}$, Lingbin Meng ${ }^{4}$, \\ Ying $\mathrm{Xin}^{5}$, Lihua Dong ${ }^{1,2,3}$, Xin Jiang ${ }^{1,2,3}$ \\ ${ }^{1}$ Department of Radiation Oncology, The First Hospital of Jilin University, Changchun, China; ${ }^{2}$ Jilin Provincial Key Laboratory of Radiation \\ Oncology \& Therapy, The First Hospital of Jilin University, Changchun, China; ${ }^{3}$ NHC Key Laboratory of Radiobiology, School of Public Health, \\ Jilin University, Changchun, China; ${ }^{4}$ Department of Hematology and Medical Oncology, Moffitt Cancer Center, Tampa, FL, USA; Key Laboratory \\ of Pathobiology, Ministry of Education, Jilin University, Changchun, China \\ \#These authors contributed equally to this work. \\ Correspondence to: Prof. Xin Jiang, PhD; Prof. Lihua Dong, PhD. Department of Radiation Oncology, the First Hospital of Jilin University, \\ 71 Xinmin Street, Changchun 130021, China. Email: jiangx@jlu.edu.cn; dlh@jlu.edu.cn.
}

\begin{abstract}
Metastatic unresectable malignant melanoma ( $M M)$ owing to its intrinsic biological invasion potential and low sensitivity to radiochemotherapy has a poor prognosis and a high rate of mortality; the mean survival period is only 6-8 months, and the 5-year survival rate is less than $10 \%$. The progression of patients with brain and liver metastases is worse than those with other distant or visceral metastases. With the advent of immunotherapy, especially immune-checkpoint inhibitors, long-term remission of stage IV disease may be achieved in some patients. Despite recent advances, not all patients benefit or can afford immunotherapy. Here, we report the case of a 44-year-old man whose initial diagnosis was MM with liver and multiple brain metastases. A high expression of F-box/WD repeat-containing protein 7 (FBXW7) inactivating mutation was observed, and the patient was treated with a combination of everolimus and temozolomide (TMZ) following palliative radiotherapy. The patient was stable for approximately 17 months, and eventually showed an overall survival (OS) of about 19 months. This case is novel and instructional, which highlights that the combination of everolimus and TMZ might be effective, with manageable toxicity, for advanced MM patients with FBXW7 mutation. And it may provide a reference for the treatment of analogous patients.
\end{abstract}

Keywords: Metastatic; malignant melanoma (MM); FBXW7; everolimus; temozolomide (TMZ)

Submitted Aug 12, 2020. Accepted for publication Nov 23, 2020.

doi: 10.21037/apm-20-1590

View this article at: http://dx.doi.org/10.21037/apm-20-1590

\section{Introduction}

Malignant melanoma (MM) is one of the most aggressive and therapy-resistant cancer that is derived from the melanocytes or cells developing from the melanocytes. The incidence of $M M$ has risen dramatically. It is estimated that about 91,270 new individuals will be diagnosed with MM, and approximately 9,320 patients will die in 2018 (1). MM has a high propensity to metastasize to the distant lymph nodes, lungs, liver, bone, and brain (2). Once MM metastasizes owing to its intrinsic biological invasion potential and low sensitivity to radiochemotherapy, prognosis is poor with a median survival of only 6 to 8 months. The progression of patients with brain and liver metastases is worse than those with other distant or visceral metastases $(2,3)$. In the past decades, it was reported that more than one-third of patients with advanced MM have brain metastases at diagnosis, and up to $75 \%$ have brain metastases at the time of death (3). It has been shown that 

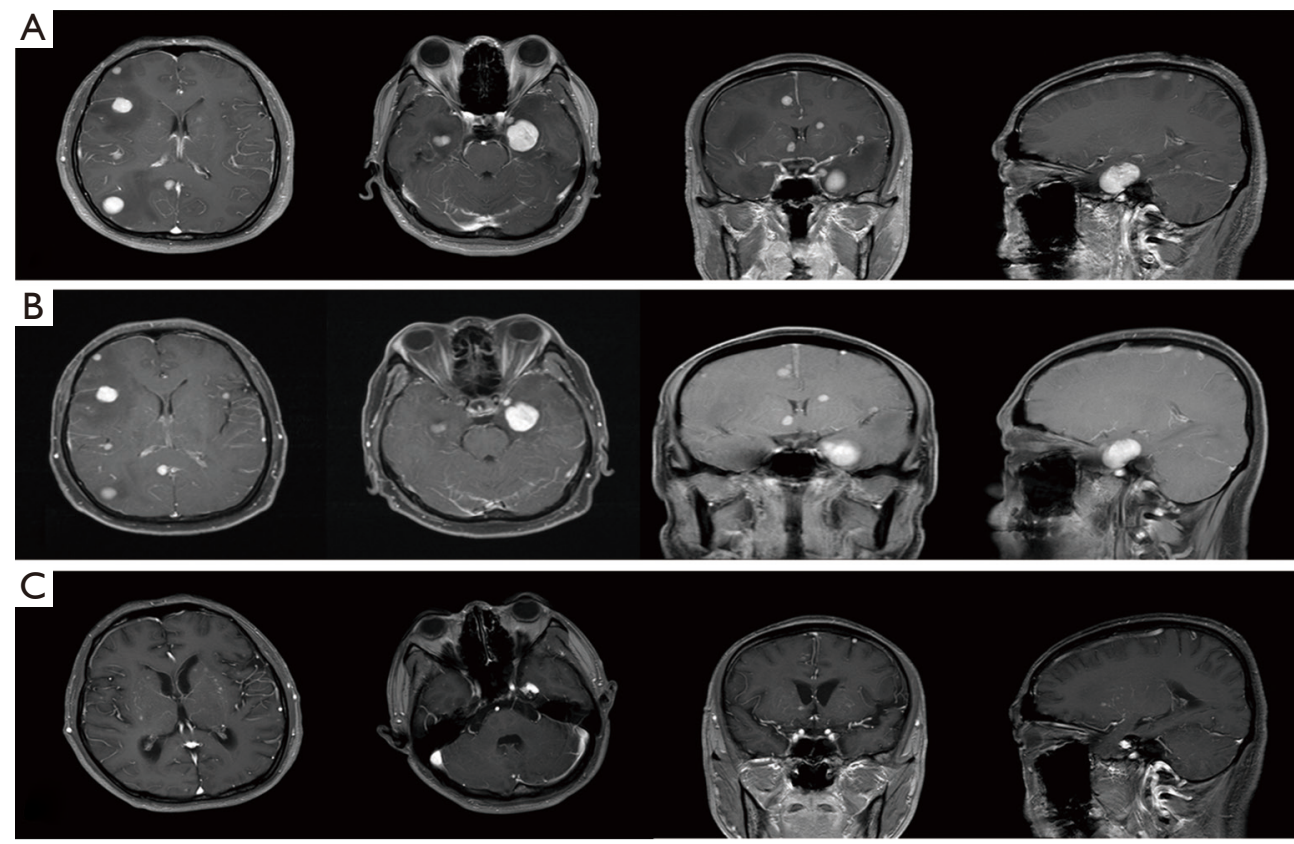

Figure 1 Magnetic resonance imaging (MRI) of brain. (A) Multiple metastases of brain at the first diagnosis. (B) At post-radiotherapy assessment: sites of brain metastases were stable. (C) At the 17-month assessment: the brain metastases had significantly shrunk.

the median survival after WBRT was only $2-4$ months. Liver metastasis may occur in approximately $50-80 \%$ of patients with advanced MM with a median survival time of only 2-6 months and a 1-year survival rate of $13 \%$ even after active treatment $(4,5)$. Here, we report the case of $M M$ with liver and multiple brain metastases. The patient was treated with a combination of everolimus and temozolomide (TMZ) that resulted in an unexpectedly prolonged survival. Our patient's outcome indicates that the combination of everolimus and TMZ could be a promising option for the clinical treatment of metastatic MM patients with FBXW7 mutation. We present the following case in accordance with the CARE reporting checklist (available at http://dx.doi. org/10.21037/apm-20-1590).

\section{Case presentation}

A 44-year-old Chinese man was admitted to the First Hospital of Jilin University for headache, vomiting, unstable gait, and declined hearing in his left ear visited our hospital 1 month in November 2016. Past medical history was as follows: three months ago, the patient underwent surgical excision of a progressively enlarged black patch with irregular borders in the occipital region of the scalp. However, no biopsy of the patch was performed after surgery. No cancer history was identified in his family. The physical examination revealed hearing decline in his left ear without altered mental status and neurologic symptoms. The brain magnetic resonance imaging (MRI) scans showed multiple long T1 and T2 signals in the right foreheadtemporal lobe, right caudate nucleus, left temporoparietal lobe, corona radiate, left insular lobe, and bilateral basal ganglia without midline shift; some lesions showed significant inhomogeneous enhancement with gadolinium contrast (Figure 1). The abdominal computed tomography (CT) scans revealed the presence of a lesion sized 10.8 $\times 10.1 \mathrm{~cm}$ in the junction region of the left and right lobes of the liver and multiple lymphadenopathy in the hilar region (Figure 2). The carbohydrate antigen (CA)-125 levels increased up to $215 \mathrm{U} / \mathrm{mL}$, and other serum tumor markers were within the normal range. Chest/pelvic CT scans and whole-body bone scintigraphy revealed no abnormal findings.

Following an ultrasound-guided percutaneous needle biopsy of the liver tumor, histopathology was performed. We observed epithelioid melanocyte proliferation with unclear cytoplasmic boundaries and hyperchromatic nucleus containing eosinophilic macronucleoli. Immunohistochemical analysis showed multifocal staining of melanocytic markers including S100, HMB-45, and Melan-A, which proved 

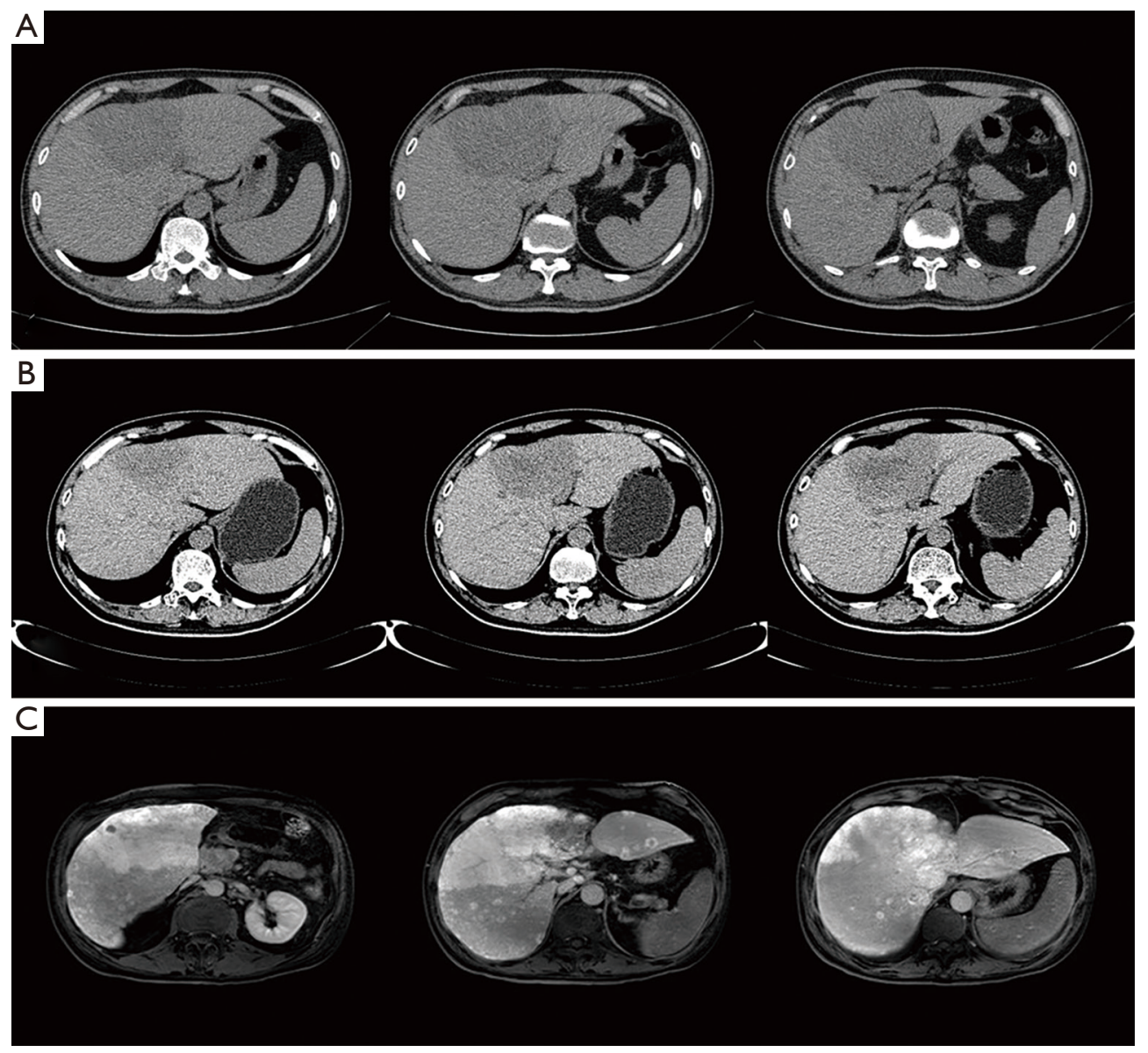

Figure 2 Computed tomography (CT) and MRI of the abdomen. (A) An occupying lesion $10.8 \times 10.1 \mathrm{~cm}$ in size in the junction region of left and right lobes of liver and multiple lymphadenopathy in hilar region; (B) at post-radiotherapy assessment: liver metastasis had slightly shrunk; (C) at the 17-month assessment: increase of liver metastases and development of pancreatic metastases.

that the tumor was an MM (Figure 3). Taken together, this patient was diagnosed as melanoma (stage IV) based on the $8^{\text {th }}$ edition of the AJCC Cancer Staging Manual (1). After multidisciplinary consultation, the liver lesion and brain metastases were considered unresectable.

The patient underwent palliative radiotherapy (56 Gy in 25 fractions) for the liver metastasis and whole-brain radiotherapy (WBRT) (40 Gy in 20 fractions) with an integrated boost of $60 \mathrm{~Gy}$ for the brain metastases and was concomitantly treated with TMZ $\left(75 \mathrm{mg} / \mathrm{m}^{2}\right.$ per day). The headache symptoms improved after radiotherapy. The brain MRI scans revealed that the metastatic sites were stable (Figure 1B). Abdominal CT scans showed a slight reduction in the volume of liver metastases (Figure 2B). No new metastases were identified. After obtaining informed consent from the patient, we performed a high-throughput sequencing analysis of the liver biopsy specimens and identified a deleterious FBXW7 p.R505C mutation, where the FBXW7 protein was inactivated. The patient could not afford the expensive immune checkpoint inhibitors. Consequently, he received treatment with oral everolimus (10 mg/day) in combination with TMZ $\left(150-200 \mathrm{mg} / \mathrm{m}^{2}\right.$ for 5 days every 28 days) starting from February 2017. The treatment was well tolerated, and the patient was generally asymptomatic but experienced inappetence and nausea. After 17 months, the brain MRI scan showed that the metastatic sites had significantly shrunk (Figure 1C); however, progression of liver disease and pancreatic metastases were detected in the abdominal MRI scan (Figure 2C). Everolimus and TMZ were suspended, and the patient received palliative treatment. The patient died 19 months after the initial diagnosis due to liver failure and 

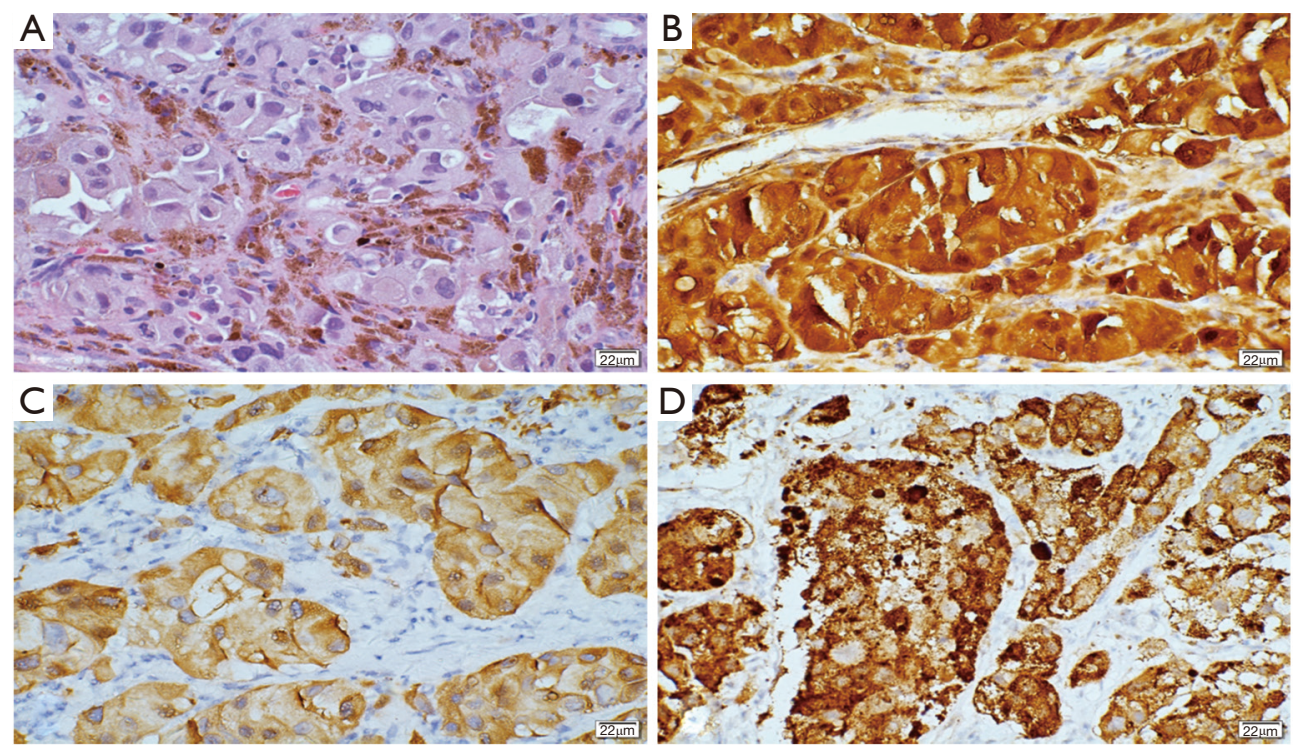

Figure 3 Histopathology of liver metastases. (A) H\&E staining: epithelioid melanocytes proliferation with unclear cytoplasmic boundaries and hyperchromatic nucleus containing eosinophilic macronucleoli (magnification $\times 100$ ). Immunohistological staining showing tumor positivity for (B) S100, (C) HMB-45, and (D) Melan-A (magnification $\times 100$ ).

disease progression.

All procedures performed in studies involving human participants were in accordance with the ethical standards of the institutional and/or national research committee(s) and with the Helsinki Declaration (as revised in 2013). Written informed consent was obtained from the patient.

\section{Discussion}

We presented the case of an advanced stage MM male patient with liver and multiple brain metastases at the initial diagnosis, and the prognoses was poor. Treatment of patients with unresectable metastatic MM is challenging; systemic therapies or clinical trial is commonly recommended. The effectiveness of the use of multiple conventional cytotoxic agents both as monotherapy or combination therapy has been evaluated in the treatment of MM; however, no significant or limited survival benefit has been observed. Adding immunostimulatory cytokines, such as INF- $\alpha$ or IL-2, to traditional cytotoxic agents has also failed to improve the overall survival (OS), but has significantly increased toxicity (6).

TMZ is converted into dacarbazine in vivo. Compared with dacarbazine, TMZ has the advantages of oral administration, not being metabolized in the liver, and penetrating the blood-brain barrier (7). Compared with
TMZ alone, WBRT combined with TMZ may prolong the survival of patients with $M M$ and unresectable brain metastases (8). Therefore, TMZ was used in combination with WBRT to alleviate the symptoms of this patient.

In the last few years, the OS of patients with metastatic MM has significantly improved with the development of personalized, targeted therapies [BRAF-V600 inhibitors, mitogen-activated protein kinase (MEK) inhibitors, and c-Kit inhibitors] and immune checkpoint inhibitors (antiPD1 and anti-CTLA4 monoclonal antibodies) $(9,10)$. High-throughput sequencing analysis of liver biopsy specimens revealed F-box/WD repeat-containing protein 7 (FBXW7) inactivating mutation; however, we did not detect other genomic mutations such as BRAFV600, MEK, or c-Kit mutations. Recent data revealed that FBXW7, a tumor-suppressor gene, encodes numerous F-box proteins. The F-box protein is a subunit of an E3-ubiquitin protein ligase complex named SKP1-cullin-F-box (SCFs), and is responsible for ubiquitination and degradation of several oncoproteins (11). The inactivating mutation of FBXW7 has been considered to be related to genetic instability, tumor proliferation, and metastasis (12). A study showed FBXW7 is a novel driver of a subset of melanoma, and this mutation was not associated with RAS or BRAF mutation (13). FBXW7 mediates the degradation of the mammalian target of rapamycin (mTOR), which plays a 
major role in tumor proliferation, angiogenesis, protein synthesis, and cell cycle progression (14). Inactivating mutation of FBXW7 increases the levels of total and activated mTOR. Preclinical studies have shown that inactivating mutation of FBXW7 can predict sensitivity to mTOR inhibitors, which are used in the treatment of MM. In this case, the patient could not afford the expensive immune checkpoint inhibitors. The patient received a combination of everolimus and TMZ according to the test results. The treatment was well tolerated for about 18 months.

The rapid development of chemotherapy resistance is the main reason for treatment failure in advanced MM. mTOR inhibitor monotherapy has no or only a slight effect on melanoma cell growth (15). Recent research showed that mTOR inhibitors such as rapamycin and its derivatives, namely temsirolimus and everolimus, can enhance the chemosensitivity of metastatic MM to TMZ. TMZ has a synergistic effect on drug-induced tumor apoptosis and reduces VEGF secretion from the tumor cells, thereby inhibiting angiogenesis (16). Treatment with a combination of mTOR inhibitors and TMZ may increase the expression of prostate transmembrane protein androgen-induced 1 (PMEPA1), early growth response protein 1 (EGR1), and Dickkopf-1 (DKK1) in melanoma cells, and consequently, induce apoptosis (17). Sinnberg et al. (18) revealed that mTOR inhibitors partly suppressed myeloid leukemia cell differentiation protein-1 (Mcl-1), a member of the antiapoptotic Bcl-2 family; this enhanced chemosensitivity to TMZ and significantly inhibited the growth of the melanoma cells (up to $60 \%$ ). A single-arm phase II multiinstitution trial suggested that the combined treatment with everolimus and $\mathrm{TMZ}$ was well tolerated in patients with advanced MM. However, the median progression-free survival was 2.4 months and the median overall survival was 8.6 months, which failed to achieve its primary endpoint for promising clinical activity (19).

In conclusion, our case suggests that somatic gene mutation analysis of MM may give us a better understanding of the genetic changes in melanoma cells. FBXW7, a tumor-suppressor gene, is a potential novel driver for MM. Routine screening of MM for FBXW7 mutation may help to explore new therapeutic strategies for the patients. And the combination of everolimus and TMZ was well tolerated and effective in the treatment of a patient metastatic unresectable MM with FBXW7 inactivating mutation. The patient was stable for approximately 17 months and eventually survived for about 19 months after being diagnosed with at an advanced stage. This is remarkable given that the mean survival period of patients with $M M$ is only 6-8 months. Considering everolimus has multiple sensitizing effects on TMZ, everolimus combined with TMZ may be a viable treatment option for patients with metastatic MM patients and FBXW7 mutation. However, further investigation in more clinical trials into their use is needed.

\section{Acknowledgments}

We would like to thank Editage (www.editage.cn) for English language editing.

Funding: This work was supported in part by grants from the National Natural Science Foundation of China (81570344, to Ying Xin), the Education Department Foundation of Jilin Province (JJKH20201036KJ); the Health and Family Planning Commission of Jilin Province Foundations (2016Q034 and 2017J11 to Ying Xin), National Key R\&D Program of China (2017YFC0112100, to Xin Jiang), the Jilin Provincial Science and Technology Foundations (20180414039GH to Ying Xin and 20190201200JC to Xin Jiang).

\section{Footnote}

Reporting Checklist: The authors have completed the CARE reporting checklist. Available at http://dx.doi.org/10.21037/ apm-20-1590

Conflicts of Interest: All authors have completed the ICMJE uniform disclosure form (available at http://dx.doi. org/10.21037/apm-20-1590). The authors have no other conflicts of interest to declare.

Etbical Statement: The authors are accountable for all aspects of the work in ensuring that questions related to the accuracy or integrity of any part of the work are appropriately investigated and resolved. All procedures performed in studies involving human participants were in accordance with the ethical standards of the institutional and/or national research committee(s) and with the Helsinki Declaration (as revised in 2013). Written informed consent was obtained from the patient.

Open Access Statement: This is an Open Access article distributed in accordance with the Creative Commons Attribution-NonCommercial-NoDerivs 4.0 International 
License (CC BY-NC-ND 4.0), which permits the noncommercial replication and distribution of the article with the strict proviso that no changes or edits are made and the original work is properly cited (including links to both the formal publication through the relevant DOI and the license). See: https://creativecommons.org/licenses/by-nc-nd/4.0/.

\section{References}

1. Smith RA, Andrews KS, Brooks D, et al. Cancer screening in the United States, 2018: A review of current American Cancer Society guidelines and current issues in cancer screening. CA Cancer J Clin 2018;68:297-316.

2. Duncan LM. The classification of cutaneous melanoma. Hematol Oncol Clin North Am 2009;23:501-13, ix.

3. Tawbi HA, Forsyth PA, Algazi A, et al. Combined Nivolumab and Ipilimumab in Melanoma Metastatic to the Brain. N Engl J Med 2018;379:722-30.

4. Rizell M, Mattson J, Cahlin C, et al. Isolated hepatic perfusion for liver metastases of malignant melanoma. Melanoma Res 2008;18:120-6.

5. Yuki T, Hori S, Yamashiro K, et al. Transarterial Chemoembolization for Liver Metastasis of Ovarian Malignant Melanoma Resulting in Long-Term Survival. Gan To Kagaku Ryoho 2016;43:1757-60.

6. Lui P, Cashin R, Machado M, et al. Treatments for metastatic melanoma: synthesis of evidence from randomized trials. Cancer Treat Rev 2007;33:665-80.

7. Goyal G, Silberstein PT. Systemic Therapy in Metastatic Melanoma. Federal practitioner : for the health care professionals of the VA, DoD, and PHS 2015;32:57S-65S.

8. Hofmann M, Kiecker F, Wurm R, et al. Temozolomide with or without radiotherapy in melanoma with unresectable brain metastases. J Neurooncol 2006;76:59-64.

9. Davey RJ, van der Westhuizen A, Bowden NA. Metastatic melanoma treatment: Combining old and new therapies.
Crit Rev Oncol Hematol 2016;98:242-53.

10. Luke JJ, Flaherty KT, Ribas A, et al. Targeted agents and immunotherapies: optimizing outcomes in melanoma. Nat Rev Clin Oncol 2017;14:463-82.

11. Yeh CH, Bellon M, Nicot C. FBXW7: a critical tumor suppressor of human cancers. Mol Cancer 2018;17:115.

12. Davis RJ, Welcker M, Clurman BE. Tumor suppression by the Fbw7 ubiquitin ligase: mechanisms and opportunities. Cancer cell 2014;26:455-64.

13. Aydin IT, Melamed RD, Adams SJ, et al. FBXW7 mutations in melanoma and a new therapeutic paradigm. J Natl Cancer Inst 2014;106:dju107.

14. Manning BD. Game of TOR - The Target of Rapamycin Rules Four Kingdoms. N Engl J Med 2017;377:1297-9.

15. Thallinger C, Werzowa J, Poeppl W, et al. Comparison of a treatment strategy combining CCI-779 plus DTIC versus DTIC monotreatment in human melanoma in SCID mice. J Invest Dermatol 2007;127:2411-7.

16. Dormond O, Contreras AG, Meijer E, et al. CD40induced signaling in human endothelial cells results in mTORC2- and Akt-dependent expression of vascular endothelial growth factor in vitro and in vivo. J Immunol 2008;181:8088-95.

17. Niessner H, Kosnopfel C, Sinnberg T, et al. Combined activity of temozolomide and the mTOR inhibitor temsirolimus in metastatic melanoma involves DKK1. Exp Dermatol 2017;26:598-606.

18. Sinnberg T, Lasithiotakis K, Niessner H, et al. Inhibition of PI3K-AKT-mTOR signaling sensitizes melanoma cells to cisplatin and temozolomide. J Invest Dermatol 2009;129:1500-15.

19. Dronca RS, Allred JB, Perez DG, et al. Phase II study of temozolomide (TMZ) and everolimus (RAD001) therapy for metastatic melanoma: a North Central Cancer Treatment Group study, N0675. Am J Clin Oncol 2014;37:369-76.
Cite this article as: Wang B, Qu C, Zhao Q, Wang H, Wei J, Meng L, Xin Y, Dong L, Jiang X. Prolonged survival following everolimus combined with temozolomide for metastatic malignant melanoma with FBXW7 mutation: a case report and literature review. Ann Palliat Med 2021;10(7):8340-8345. doi: 10.21037/apm-20-1590 